\title{
Mujeres que usan drogas inyectadas y violencia: Necesidad de una respuesta integrada
}

\author{
Women who inject drugs and violence: \\ Need for an integrated response
}

\author{
Cinta Folch*,**, Jordi Casabona*,**, Xavier Majó***, Mercè Meroño****, Victoria \\ González*,*****, Joan Colom***, M. Teresa Brugal**,******, Albert Espelt**,******,*******. \\ * Centre d'Estudis Epidemiològics sobre les Infeccions de Transmissió Sexual i Sida de Catalunya (CEEISCAT), \\ Dept. Salut. Generalitat de Catalunya. España. \\ ** Centro de Investigación Biomédica en Red de Epidemiología y Salud Pública (CIBERESP), Madrid. España. \\ *** Sub-direcció General de Drogodependències, Agència de Salut Pública de Catalunya. Programa de Prevenció, \\ Control i Atenció al VIH, les ITS i les Hepatitis Víriques (PCAVIHV), Barcelona. España. \\ **** Fundació Àmbit Prevenció, Barcelona. España. \\ ***** Servicio de Microbiología. Laboratori Clínic de la Metropolitana Nord. Hospital Universitari Germans \\ Trias i Pujol, Badalona. España. \\ ******* Agència de Salut Pública de Barcelona, Barcelona. España. \\ ******* Facultat de Ciències de la Salut de Manresa. Universitat de Vic Universitat Central de Catalunya (UVicUCC), \\ Manresa. España.
}

\section{Resumen}

El objetivo de este estudio fue describir la prevalencia de violencia física y/o sexual experimentada por mujeres que usan drogas por vía inyectada (MUDVI) e identificar factores asociados. Se realizó un estudio transversal en 120 MUDVI usuarias de centros de reducción de daños mediante un cuestionario anónimo y recogida de muestras de fluido oral para estimar la prevalencia del VIH y de la hepatitis C. Los factores asociados a la presencia de violencia se analizaron mediante un modelo de regresión de Poisson con varianza robusta univariante y multivariante, obteniendo razones de prevalencia (RP) y sus intervalos de confianza al 95\%. Los resultados muestran que la prevalencia de agresiones en los últimos 12 meses fue del 45,8\% (42,2\% agresiones físicas y 11,9\% agresiones sexuales). A nivel multivariante, las variables asociadas a la presencia de violencia fueron estar sin domicilio fijo ( $\mathrm{RP}=1,59$; IC: 1,07-2,38), ejercer el trabajo sexual ( $\mathrm{RP}=1,65$; IC: 1,19$2,29)$, haber sufrido alguna infección de transmisión sexual $(\mathrm{RP}=1,49$; IC: $1,04-2,15)$ y/o inyectarse drogas no de forma diaria $(\mathrm{RP}=2,29$; IC: 1,49-3,54). Este estudio pone de manifiesto la importancia de establecer protocolos de detección, y derivación a la red de atención a la violencia de género, dentro de los centros de la red de atención a las drogodependencias, así como el desarrollo de estrategias multinivel que tengan en cuenta no solamente factores individuales sino también otros aspectos sociales y/o estructurales que pueden estar jugando un papel relevante a la hora de abordar este problema. Palabras clave: Centros de reducción de daños; hepatitis C; mujeres; uso de drogas inyectadas; VIH; violencia.

\begin{abstract}
The aim of this study was to describe the prevalence of physical and/ or sexual violence experienced by women who inject drugs (WWID) and identify associated factors. A cross-sectional study was conducted among 120 WWID in a network of harm reduction centres using an anonymous questionnaire. Oral fluid samples were also collected to estimate the prevalence of HIV and hepatitis C. Univariate and multivariate Poisson regression models with robust variance were performed to identify the factors associated with experiencing violence, obtaining prevalence ratios (PR) and their 95\% confidence intervals. The results showed that the prevalence of violence reported by WWID in the last 12 months was $45.8 \%$ (42.2\% physical and $11.9 \%$ sexual aggression). In multivariate analysis, variables associated with experiencing violence were homelessness ( $\mathrm{PR}=1.59$; CI: 1.07-2.38), reporting exchanges of sex for money or drugs $(\mathrm{PR}=1.65$; CI: 1.19 2.29), reporting a previous sexually transmitted infection ( $\mathrm{PR}=$ 1.49; CI: 1.04-2.15) and/or injecting drugs less frequently than daily $(\mathrm{RP}=2.29$; CI: 1.49-3.54). This study highlights the importance of establishing detection protocols and systems of referral to the network of attention to women suffering violence, within the centres of the drug addiction care network, as well as the development of multilevel strategies that take into account not only individual factors but also other social and/or structural aspects that may be playing a relevant role in addressing this problem.

Keywords: Harm reduction centres; hepatitis C; women; drug injection; HIV; violence.
\end{abstract}




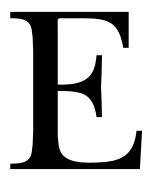

n general, la investigación, prevención y tratamiento de las adicciones se contempla desde un punto de vista androcéntrico, es decir, con una visión centrada en el punto de vista masculino. Las mujeres han sido invisibilizadas en este fenómeno, y por tanto en las respuestas o políticas para abordarlo (Jiménez, Molina y García-Palma, 2014). La estigmatización y el rechazo social que soportan las mujeres con problemas de adicción a las drogas, y en particular las mujeres que usan drogas por vía inyectada (MUDVI), se traduce en un menor apoyo social o familiar, mayor aislamiento y ausencia y/o demora en la solicitud de ayuda para superar el problema (Arpa, 2017; Falcón, 2006; UN Women, 2014). Según ONUSIDA, 48 países reportan datos de prevalencia de VIH desagregando por sexo desde el año 2011, con diferencias importantes según zona geográfica en las estimaciones de prevalencia del VIH en MUDVI. Por otro lado, en la mayoría de estos países (28 de 41) la prevalencia de VIH observada en mujeres es superior que la reportada en hombres que usan drogas inyectadas (UNAIDS, 2014). En Cataluña, la prevalencia de VIH estimada en MUDVI reclutadas en centros de reducción de daños durante 2008-2011 fue también superior a la observada en hombres $(38,7 \%$ y $31,5 \%$, respectivamente) (Folch et al., 2008).

Entre los factores que la literatura describe asociados a la mayor vulnerabilidad que presentan las MUDVI a la infección por VIH, la Hepatitis C, y otras Infecciones de Transmisión Sexual (ITS) destacan factores relacionados con el propio individuo (baja autoestima, soledad, prácticas de riesgo), factores sociales y/o comunitarios (problemas de consumo en la familia, conflictos de pareja), y factores a nivel estructural (discriminación, leyes y políticas) (Baral, Logie, Grosso, Wirtz y Beyrer, 2013; El-Bassel, Wechsberg y Shaw, 2012). Uno de estos factores descrito en la literatura de forma recurrente es la violencia física y/o sexual que las MUDVI experimentan, principalmente de sus parejas sexuales, y que puede reducir su capacidad de proteger su propia salud negociando prácticas sexuales y de inyección más seguras (Azim, Bontell y Strathdee, 2015; Iversen, Page, Madden y Maher, 2015). Por otro lado, los prejuicios y el estigma social que recaen sobre las mujeres consumidoras de drogas, ya que no cumplen con las expectativas del ideal socialmente aceptado, limitan y/o retrasan el acceso a los centros de tratamiento y rehabilitación (Malinowska-Sempruch, Rychkova y Foundations, 2015).

En los últimos años diversos autores han puesto de manifiesto la interacción sinérgica que aparece al combinar abuso de sustancias, violencia de género y la infección por VIH bajo el concepto de sindemia SAVA (Substance Abuse, Violence and AIDS) (Gilbert et al., 2015; Meyer, Springer y Altice, 2011). Aunque la violencia de género que se ejerce sobre las mujeres, que incluye el abuso sexual infantil, la violencia de pareja, y la agresión sexual
(UN General Assembly, 1993), no es un mecanismo directo para la transmisión del VIH, un meta análisis reciente indica que puede causar un incremento del riesgo de VIH entre $28-52 \%$ entre las diferentes poblaciones de mujeres, incluyendo el colectivo de mujeres que usan drogas (Li et al., 2014). Las consecuencias psicológicas asociadas a la violencia que sufren estas mujeres, como ansiedad, depresión y baja autoestima, puede limitar su capacidad de realizar prácticas de inyección y sexuales más seguras (Wagner et al., 2009). Por otro lado, la violencia en mujeres con problemas de adicción a las drogas se transforma en dificultad de acceso y permanencia en el tratamiento, sumado al hecho de que tanto desde la red de atención a las mujeres víctimas de violencia de género, como desde la red de atención a las drogodependencias, ha sido difícil incorporar la doble visión de la drogodependencia y la violencia en los protocolos de atención (Lipsky et al., 2010; Ruiz-Olivares y Chulkova, 2016), a pesar de la evidencia que algunos trabajos muestran de intervenciones psicosociales para abordar violencia de pareja en mujeres que usan drogas en los centros de tratamiento de drogas (Gilbert et al., 2006; Tirado-Muñoz, Gilchrist, Lligoña, Gilbert y Torrens, 2015.)

En este sentido, en España existen escasos estudios que cuantifiquen y/o aborden específicamente la violencia en mujeres drogodependencias y sus consecuencias. Un ejemplo de estos estudios es el realizado en Cataluña en centros de tratamiento de drogas en el que no se observaron diferencias en cuanto a los niveles de violencia sufridos por hombres y mujeres que acudían a estos centros, aunque si en función del tipo de violencia sufridos, siendo más común la violencia psicológica y sexual entre las mujeres (Arribas-Ibar et al., 2018). Es por tanto importante aportar elementos que puedan ayudar al diseño de acciones apropiadas y basadas en la evidencia para el manejo integral de la violencia de género y las drogodependencias. En este sentido, el objetivo de este estudio fue describir la prevalencia de violencia física y/o sexual experimentada por MUDVI que acuden a centros de reducción de daños en Cataluña e identificar factores asociados a haber sufrido algún acto de violencia en los últimos 12 meses.

\section{Métodos}

Estudio descriptivo transversal realizado en 2014-15 en MUDVI usuarias de centros de reducción de daños en Cataluña como parte del Sistema Integrado de Vigilancia Epidemiológica del VIH/ITS de Cataluña. Los programas de reducción de daños en estos centros incluyen programas de intercambio de jeringas (PIJ), programas de acercamiento (outreach), centros de atención y seguimiento de las drogodependencias (CAS) y salas de consumo supervisado. 


\section{Participantes}

Se seleccionó una muestra de conveniencia de MUDVI estratificando por centro y por área geográfica de origen. La asignación al estrato se hizo proporcionalmente al volumen de visitas de cada centro y al porcentaje de individuos según área geográfica de origen. Previamente se llevó a cabo un estudio de prospección o mapeo para poder conocer el tipo de población que acude en cada centro. Los criterios de inclusión fueron ser mayor de 18 años y haberse inyectado drogas alguna vez en los últimos 6 meses.

\section{Cuestionario}

Se recogió la información epidemiológica y conductual mediante un cuestionario anónimo administrado por entrevistadores, traducido al castellano, rumano, y ruso, y adaptado del utilizado por la OMS (WHO, 1994). El cuestionario recogía información sobre características sociodemográficas, patrones de consumo de drogas, conductas de riesgo relacionadas con la inyección, comportamiento sexual, conocimiento del estado serológico frente al VIH, VHC y otras ITS, uso de servicios sociales y sanitarios, estancia en prisión, acceso a prevención y violencia sufrida en el último año, entre otras. En concreto, las preguntas sobre violencia fueron: "En los últimos 12 meses, ¿̇cuántas veces has recibido algún tipo de agresión física (te han pegado, empujado, golpeado,...)?, así como, En los últimos 12 meses, ¿̇cuántas veces has recibido algún tipo de agresión o abuso sexual? La variable dependiente se definió como haber sufrido algún acto de violencia física y/o agresión o abuso sexual en los últimos 12 meses.

\section{Muestras biológicas}

Por otro lado, se recogieron muestras de fluido oral utilizando el instrumento ORASURE (Epitope Inc. USA) para estimar la prevalencia de anticuerpos frente a la infección por el VIH y VHC, respectivamente. Para la detección de anticuerpos anti-VIH en muestras de fluido oral se utilizaron los kits Detec-HIV versión 4 de ADALTIS (Chohan et al., 2001) y para la detección de anticuerpos anti-VHC el ensayo HCV 3.0 SAVe ELISA (Ortho-Clinical Diagnostics) (Judd et al., 2003). Al ser un estudio anónimo y al realizarse un test válido para estudio epidemiológico pero no aprobado para diagnóstico individual, a los participantes no se les entregaba el resultado de los tests, pero se les ofrecía la posibilidad de realizarse las pruebas rápidas en la red de centros alternativos de oferta de la prueba en Cataluña.

\section{Aspectos éticos}

Todas las participantes firmaron un consentimiento informado. El protocolo del estudio fue aprobado por el Comité de Ética del Hospital Universitari Germans Trias i Pujol.

\section{Análisis estadístico}

Se realizó un análisis descriptivo de las principales variables y se compararon las proporciones según si habían sufrido situaciones de violencia o no mediante la prueba de la $\chi 2$ de Pearson y el test Exacto de Fisher. Las variables cuantitativas se compararon mediante la prueba t para muestras independientes después de realizar prueba de Levene para la igualdad de varianzas. Los factores asociados a haber sufrido violencia sexual y/o física en mujeres se analizaron mediante un modelo de regresión de Poisson con varianza robusta univariante y multivariante (Espelt, Bosque-Prous y Marí-Dell'Olmo, 2019; Espelt, Mari-Dell'Olmo, Penelo y Bosque-Prous, 2017), estimando Razones de Prevalencia (RP) y su intervalo de confianza (IC) del 95\%. Variables con un nivel de significación $<0,10$ en el análisis univariante se incluyeron en el modelo multivariante. Para todos los análisis se consideró un nivel de error del $5 \%$ y se utilizó el paquete estadístico SPSS versión 17.

\section{Resultados}

Del total de 120 MUDVI que participaron en el estudio, más de la mitad eran de origen español $(67,2 \%)$ y el $32,8 \%$ eran originarias de otros países, principalmente del este de Europa (42,9\%) e Italia (35,7\%). La edad media fue de 35,4 años (DE: 8,9; rango: 18-61 años) y el 50,8\% de las mujeres estaba en la actualidad en tratamiento por su adicción.

La prevalencia de agresiones en los últimos 12 meses fue del $45,8 \%$ en total, del 42,2\% en el caso de agresiones físicas y del $11,9 \%$ en el caso de agresiones sexuales. Un 8,3\% de las MUDVI reportaron haber sufrido ambos tipos de agresiones. Entre las MUDVI que afirmaron haber sufrido algún tipo de agresión física en los últimos 12 meses ( $n=51$ ), un $37,3 \%$ fue en una ocasión, $23,5 \%$ en dos y el resto en tres o más ocasiones $(39,2 \%)$. En el caso de las 15 mujeres que han sufrido agresiones sexuales, el 66,7\% afirma que fue en una sola ocasión, $20 \%$ dos ocasiones y $13,4 \%$ tres o más ocasiones.

En la tabla 1 se describe el perfil socio demográfico y conductual de las mujeres según si han sufrido algún tipo de agresión física y/o sexual o no. Se observan diferencias estadísticamente significativas en el porcentaje de MUDVI que refirieron haber mantenido relaciones sexuales comerciales, siendo este porcentaje superior entre las MUDVI que afirmaron haber sufrido algún tipo de agresión en los últimos 12 meses (29,1\% y 9,7\%, respectivamente).

La prevalencia autodeclarada de ITS (alguna vez en la vida) fue superior en MUDVI que afirmaron haber sufrido algún tipo de violencia (49,1\% (IC: 36,4\%-61,9\%) frente 24,6\% (IC: $15,8 \%-36,3 \%$ ); $\mathrm{p}<0,001$ ). No se observaron diferencias significativas en la prevalencia del VIH y del VHC entre las mujeres que afirmaron haber sufrido algún tipo de violencia y las que no (Figura 1). 
Tabla 1. Perfil socio demográfico, patrones y conductas de consumo de drogas y sexuales en MUDVI según si han sufrido o no agresiones (últimos 12 meses).

\begin{tabular}{|c|c|c|c|}
\hline & \multicolumn{2}{|c|}{$\begin{array}{c}\text { Violencia física y/o } \\
\text { sexual }\end{array}$} & \multirow[b]{2}{*}{$\mathbf{p}$} \\
\hline & $\begin{array}{c}\text { No }(n=65) \\
\%\end{array}$ & $\begin{array}{c}\mathrm{Si}(\mathrm{n}=55) \\
\%\end{array}$ & \\
\hline Edad: Menos de 30 años & $19(29,2)$ & $18(32,7)$ & 0,679 \\
\hline País de origen: España & $42(64,6)$ & $47(67,3)$ & 0,094 \\
\hline Nivel de estudios primarios o inferior & $42(64,6)$ & $35(63,6)$ & 0,911 \\
\hline Alguna vez en la cárcel & $34(52,3)$ & $38(69,1)$ & 0,061 \\
\hline En tratamiento actualmente & $32(49,2)$ & $28(50,9)$ & 0,953 \\
\hline Años de inyección:0-5 & $22(33,8)$ & $15(27,8)$ & 0,476 \\
\hline Inyección diaria o 2-3 días/semana* & $48(73,8)$ & $39(70,9)$ & 0,720 \\
\hline Ha aceptado jeringuillas usadas* & $10(15,4)$ & $10(18,2)$ & 0,682 \\
\hline Ha pasado jeringuillas usadas* & $21(32,3)$ & $18(32,7)$ & 0,961 \\
\hline Ha compartido otro material* & $41(63,1)$ & $38(70,4)$ & 0,402 \\
\hline $\begin{array}{l}\text { Relaciones sexuales con pareja } \\
\text { estable (PE)* }\end{array}$ & $40(61,5)$ & $38(69,1)$ & 0,387 \\
\hline $\begin{array}{l}\text { Relaciones sexuales con pareja } \\
\text { ocasional (PO)* }\end{array}$ & $14(21,5)$ & $15(27,3)$ & 0,465 \\
\hline $\begin{array}{l}\text { Uso no consistente del preservativo } \\
\text { con } \mathrm{PE}^{\star}\end{array}$ & $33(50,8)$ & $33(60,0)$ & 0,597 \\
\hline $\begin{array}{l}\text { Uso no consistente del preservativo } \\
\text { con PO* }\end{array}$ & $5(7,7)$ & $9(16,4)$ & 0,324 \\
\hline Relaciones sexuales con clientes* & $6(9,7)$ & $16(29,1)$ & 0,007 \\
\hline Relaciones sexuales con PE inyectora & $25(38,5)$ & $28(50,9)$ & 0,171 \\
\hline
\end{tabular}

Nota. *últimos 6 meses.
En relación a las personas que las agredieron físicamente y/o sexualmente destacan la pareja o cónyuge en primer lugar $(43,1 \%$ y $35,3 \%$, respectivamente) y los amigos y/o conocidos en segunda posición $(22,4 \%$ y $23,5 \%$, respectivamente). El $6 \%$ aproximadamente de las MUDVI que han sufrido violencia física/o sexual afirma que fue por parte de sus clientes sexuales y un $10 \%$ de las que han sufrido violencia física afirman que fue por parte de la policía (Tabla 2).

Los factores asociados a nivel univariante y multivariante con haber sufrido violencia sexual y/o física se presentan en la tabla 3. A nivel multivariante, se observa una asociación entre estar sin domicilio fijo ( $\mathrm{RP}=1,59$; IC: 1,07-2,38), ejercer el trabajo sexual ( $\mathrm{RP}=1,65$; IC: 1,19-2,29), declarar haber sufrido alguna ITS ( $\mathrm{RP}=1,49$; IC: 1,04-2,15) y/o inyectarse drogas no de forma diaria con la presencia de violencia en MUDVI (RP=2,29; IC: 1,49-3,54).

Tabla 2. Violencia física y/o sexual en MUDVI por tipo de autor (últimos 12 meses).

\begin{tabular}{lcc}
\hline & $\begin{array}{c}\text { Violencia física } \\
(\mathbf{n = 5 1 )} \\
\%\end{array}$ & $\begin{array}{c}\text { Violencia sexual } \\
(\mathbf{n}=\mathbf{1 5}) \\
\%\end{array}$ \\
\hline Pareja o cónyuge & 43,1 & 35,3 \\
Padres & 3,4 & 0,0 \\
Familiares & 3,4 & 0,0 \\
Amigos/conocidos & 22,4 & 23,5 \\
Clientes sexuales & 6,9 & 5,9 \\
Implicados en tráfico de drogas & 5,2 & 11,8 \\
Vecinos & 0,0 & 5,9 \\
Policía & 10,3 & 0,0 \\
Desconocidos & 25,9 & 2,4 \\
\hline
\end{tabular}

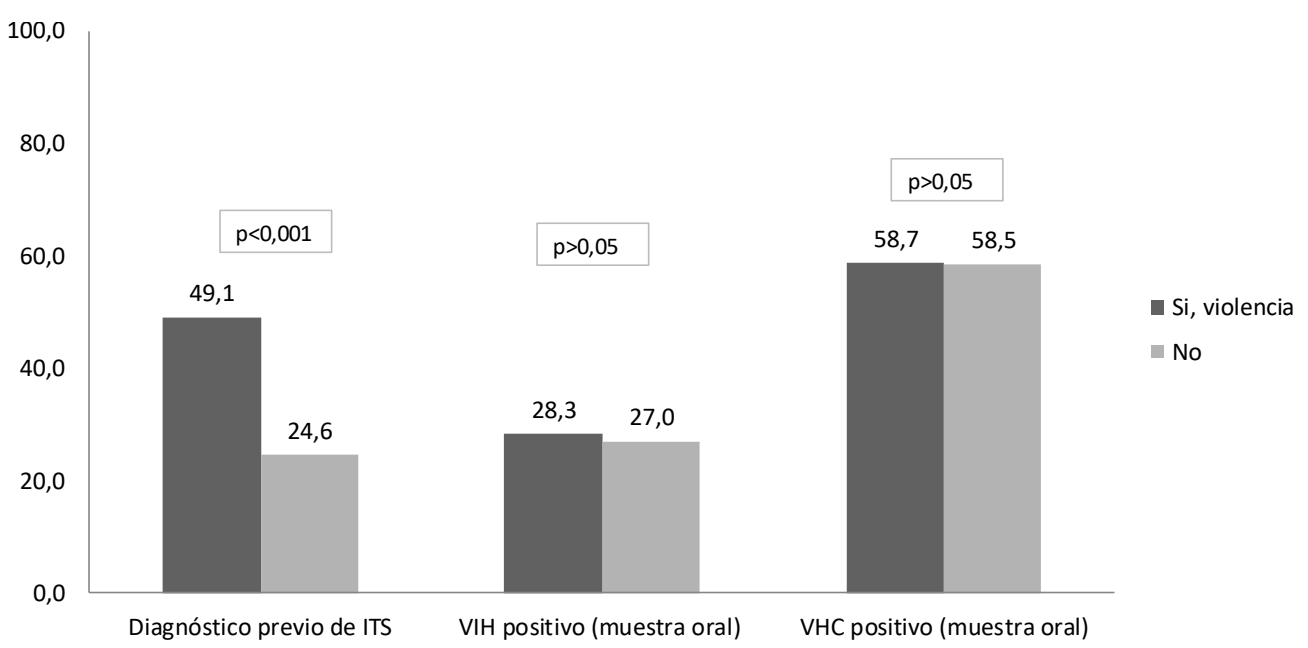

Figura 1. ITS autodeclaradas y prevalencia de VIH y Hepatitis C en MUDI según si han sufrido violencia o no. 


\section{Discusión}

Los datos de este estudio confirman la elevada prevalencia de agresiones físicas y/o sexuales que sufren las MUDVI en nuestro país, muchas de ellas por parte de sus parejas o cónyuges, prevalencia superior a la observada en mujeres en población general (Llopis, Castillo, Rebollida y Stocco, 2005). En concreto, aproximadamente la mitad de las MUDVI usuarias de centros de reducción de daños en Cataluña afirman haber sufrido este tipo de violencia en el último año, siendo esta prevalencia mayor en mujeres que

Tabla 3. Perfil socio demográfico, patrones y conducta sexual y de consumo de drogas asociadas a sufrir agresiones en MUDVI (últimos 12 meses).

\begin{tabular}{|c|c|c|c|c|c|}
\hline & $\mathbf{P}$ & $\mathbf{R P}$ & IC95\% & $\mathrm{RPa}$ & IC95\% \\
\hline \multicolumn{6}{|l|}{ Edad } \\
\hline «30 años & 48,6 & 1 & & & \\
\hline 30 años o más & 44,6 & 0,92 & $0,61-1,38$ & & \\
\hline \multicolumn{6}{|l|}{ País de origen } \\
\hline España & 46,8 & 1 & & & \\
\hline Otro & 43,9 & 0,94 & $0,62-1,43$ & & \\
\hline \multicolumn{6}{|l|}{ Nivel de estudios } \\
\hline Primaria o inferior & 45,4 & 1 & & & \\
\hline$\geq$ Secundaria & 46,5 & 1,02 & $0,68-1,53$ & & \\
\hline \multicolumn{6}{|c|}{ Alguna vez en la cárcel } \\
\hline No & 35,4 & 1 & & & \\
\hline Sí & 52,8 & 1,49 & $0,96-2,32$ & & \\
\hline \multicolumn{6}{|l|}{ Sin domicilio fijo* } \\
\hline No & 42,6 & 1 & & 1 & \\
\hline \multicolumn{6}{|l|}{ Fuente de ingresos* } \\
\hline No legal & 37,5 & 1 & & & \\
\hline Legal & 51,4 & 1,37 & $0,89-2,11$ & & \\
\hline \multicolumn{6}{|l|}{ Años de inyección } \\
\hline $0-5$ & 40,5 & 1 & & & \\
\hline$>5$ & 47,6 & 1,17 & $0,75-1,85$ & & \\
\hline \multicolumn{6}{|l|}{ Inyección diaria } \\
\hline No & 59,1 & 1,99 & $1,26-3,16$ & 2,29 & $1,49-3,54$ \\
\hline Sí & 29,6 & 1 & & 1 & \\
\hline \multicolumn{6}{|c|}{ Aceptar jeringuillas usadas* } \\
\hline No & 45,0 & 1 & & & \\
\hline Sí & 50,0 & 1,11 & $0,68-1,81$ & & \\
\hline \multicolumn{6}{|c|}{ Pasar jeringuillas usadas* } \\
\hline No & 45,7 & 1 & & & \\
\hline No & 40,5 & 1 & & & \\
\hline Sí & 48,7 & 1,2 & $0,78-1,86$ & & \\
\hline \multicolumn{6}{|l|}{ Pareja ocasional } \\
\hline No & 44,0 & 1 & & & \\
\hline Sí & 51,7 & 1,17 & $0,77-1,80$ & & \\
\hline \multicolumn{6}{|c|}{ Relaciones sexuales con clientes* } \\
\hline No & 41,1 & 1 & & 1 & \\
\hline Sí & 72,7 & 1,77 & $1,24-2,52$ & 1,65 & $1,19-2,29$ \\
\hline \multicolumn{6}{|c|}{ Pareja estable inyectora } \\
\hline No & 40,3 & 1 & & & \\
\hline Sí & 52,8 & 1,31 & $0,89-1,93$ & & \\
\hline \multicolumn{6}{|l|}{ VIH (fluido oral) } \\
\hline No & 45,2 & 1 & & & \\
\hline Sí & 46,9 & 1,04 & $0,67-1,61$ & & \\
\hline \multicolumn{6}{|l|}{ VHC (fluido oral) } \\
\hline No & 45,8 & 1 & & & \\
\hline Sí & 45,6 & 0,99 & $0,66-1,49$ & & \\
\hline \multicolumn{6}{|l|}{ Alguna ITS } \\
\hline No & 36,4 & 1 & & 1 & \\
\hline Sí & 62,8 & 1,73 & $1,19-2,52$ & 1,49 & $1,04-2,15$ \\
\hline
\end{tabular}

Nota. *últimos 6 meses; RP: Razón de prevalencia; RPa: Razón de prevalencia ajustada. 
ejercen el trabajo sexual y aquellas sin domicilio fijo, justificando así la necesidad de desarrollar estrategias de prevención combinada (UNAIDS, 2010) que tengan en cuenta no solamente factores individuales, sino también otros aspectos sociales y/o estructurales que pueden estar jugando un papel relevante a la hora de abordar este problema.

Diversos estudios realizados anteriormente ya evidenciaron una asociación entre ser mujer consumidora de drogas y una mayor probabilidad de sufrir algún tipo de violencia (Arribas-Ibar et al., 2018; Llopis et al., 2005). La mayor parte de las situaciones de violencia en MUDVI que acuden a centros de reducción de daños procede de sus parejas estables, muchas de ellas también consumidoras de droga por vía inyectada. En estas parejas se ha visto que la separación es compleja ya que se mezcla la ambivalencia ante la relación y la ambivalencia ante el consumo (Chait y Calvo, 2005). Además, muchas veces las MUDVI no identifican las situaciones de violencia hacia ellas o asumen como "normal" la mayor agresividad de sus parejas cuando están bajo los efectos de las sustancias (Martínez Redondo, 2009). Sin embargo, es un error identificar exclusivamente la violencia en el ámbito de la pareja debido a que en un elevado porcentajes estas agresiones se dan también en el entorno de amistades y conocidos, e incluso por desconocidos y en el caso de las agresiones físicas por parte de policías. Estudios previos realizados en otros países como por ejemplo Canadá también muestran un elevado porcentaje de MUDVI $(43,5 \%)$ que dicen haber sido víctimas de violencia por parte de conocidos (Marshall, Fairbairn, Li, Wood y Kerr, 2008).

Los datos del estudio muestran una asociación entre haber sufrido algún tipo de violencia en el último año y ejercer el trabajo sexual a cambio de dinero y/o drogas, resultado consistente con estudios previos realizados a nivel internacional (Azim et al., 2015). Las MUDVI que ejercen el trabajo sexual se enfrentan muchas veces con ciertas barreras sociales y estructurales que les impiden acceder a los programas de prevención y a los servicios socio-sanitarios necesarios (El-Bassel, Shaw, Dasgupta y Strathdee, 2014). Además de las situaciones de violencia a la que se enfrentan las mujeres que ejercen el trabajo sexual, especialmente las mujeres que ejercen en la calle (Deering et al., 2013), las MUDVI se enfrentan con situaciones de riesgo particularmente alto de infección por el VIH y otras ITS, como por ejemplo el consumir drogas con sus clientes (Strathdee et al., 2011), o dificultades de negociación del preservativo con clientes estables que les dan más dinero para poder financiar su consumo (Robertson et al., 2014).

Vivir en la calle se muestra también asociado con haber sufrido algún tipo de violencia en el último año, resultado ya observado en estudios previos tanto en hombres como en mujeres que consumen drogas inyectadas (Marshall et al., 2008). Las MUDVI sin hogar sufren formas agravadas de subordinación y desigualdad que refuerzan su invisibi- lidad y exclusión social, y que se expresan a veces través de la violencia, ya sea de sus parejas o de sus clientes en el caso que ejerzan el trabajo sexual (Bourgois, Prince y Moss, 2004). Esta asociación podría deberse además a distintos factores relacionados con las condiciones precarias sociales que presentan estas personas (actividades delictivas, pobreza, poco soporte social...) (Marshall et al., 2008).

Se observa una mayor prevalencia de ITS (alguna vez en la vida) en MUDVI que han sufrido violencia (49\% frente a 25\%). Aunque no exclusivo del colectivo de MUDVI, los estudios muestran un mayor riesgo de ITS/VIH en mujeres que han sufrido algún tipo de violencia sexual por parte de sus parejas (Decker et al., 2014). En este sentido, se constata un menor uso del preservativo en la relaciones sexuales debido a las dificultades de negociación del uso del preservativo cuando hay violencia de por medio. Se puede decir que el consumo de drogas, la violencia y las infecciones asociadas son problemas de salud sinérgicos que interactúan mutuamente y que afectan negativamente la salud de la población, en este caso de la población de MUDVI (Gilbert et al., 2015).

Un dato que sorprende es la menor probabilidad de haber sufrido violencia entre MUDVI que se inyectan diariamente en comparación con MUDVI que se inyectan con una menor frecuencia. Un análisis más en detalle de las características del consumo sería necesario para poder interpretar los resultados correctamente (consumo por otras vías, policonsumo, etc).

Entre las limitaciones del estudio destaca, en primer lugar, el no poder generalizar los datos al total de los MUDVI de Cataluña, ya que la muestra se ha seleccionado sólo en centros de reducción de daños, por lo que no disponemos de información del resto de mujeres que no acceden a estos centros. Sin embargo, se ha intentado diversificar al máximo el tipo de centro de reclutamiento y se han incluido personas procedentes de otros países para poder tener una muestra lo más representativa posible. Por otro lado, la prevalencia de algunas conductas de riesgo recogidas mediante el autoinforme podría estar subestimada, así como el porcentaje de mujeres que dice haber sufrido algún tipo de agresión física y/o sexual. En este sentido, se intentó establecer un ambiente de anonimato que favoreciera la realización de las entrevistas, utilizando un lenguaje simple y comprensible. Por otro lado, ciertos tipos de violencia pueden no haber sido recogidos mediante la pregunta que se incluyó en REDAN (violencia psicológica por ejemplo). Además, las propiedades psicométricas del cuestionario en el presente trabajo no fueron evaluadas. A pesar del reducido tamaño de la muestra, este trabajo es uno de los pocos estudios que existen en nuestro país que aborden el problema de las adicciones desde una perspectiva de género. Sería interesante poder llevar a cabo estudios futuros incluyendo un mayor número de participantes para poder realizar análisis con mayor poder estadístico. Por último, 
al tratarse de un estudio descriptivo transversal, no podrán establecerse relaciones de causalidad entre las situaciones de violencia y los factores de riesgo analizados.

A pesar de las limitaciones, los datos de este estudio ponen de manifiesto que un elevado porcentaje de MUDVI reclutadas en centros de reducción de daños ha sufrido violencia física y/o sexual en alguna ocasión, especialmente aquellas mujeres con peores condiciones sociales como las que declaran vivir en la calle y las que ejercen el trabajo sexual. En este sentido, se justifica la importancia de establecer protocolos de detección, y derivación a la red de atención a la violencia de género, dentro de los centros de la red de atención a las drogodependencias donde acuden estas mujeres, dada la dificultad que a veces manifiestan estas mujeres para identificar las situaciones de violencia debido a que las han integrado en su cotidiano como lo "normal".

Por otro lado, es importante también formar a los/as profesionales que atienden a las mujeres, generar espacios seguros para ellas y poder trabajar desde su seguridad, sin hombres, y también trabajar nuevas masculinidades basadas en el respeto hacia las mujeres. Finalmente, se debería de asegurar que las mujeres que consumen drogas y que además se enfrentan a situaciones de violencia puedan acceder a recursos que realicen intervenciones holísticas de atención integral a estas mujeres, ya que la mayoría de estos servicios están pensados para atender a hombres al ser la mayoría visible.

\section{Reconocimientos}

Los autores agradecen a los/as entrevistadores/as, a los centros de reducción de daños participantes, a Oleguer Parés por sus aportaciones al manuscrito, y especialmente a todas las mujeres que usan drogas que participaron en el estudio.

\section{Conflicto de intereses}

Albert Espelt es editor asociado de la revista Adicciones. Sin embargo, no ha tenido ningún papel durante el proceso editorial.

\section{Bibliografía}

Arpa, S. (2017). Women who use drugs: Issues, needs, responses, challenges and implications for policy and practice. Lisbon, Portugal: EMCDDA Papers, Publications Office of the European Union.

Arribas-Ibar, E., Suelves, J. M., Sanchez-Niubò, A., Tirado-Muñoz, J., Domingo-Salvany, A. y Brugal, M. T. (2018). Violence among illicit drug users recruited in drug treatment facilities. Adicciones. Avance de publicación on-line. doi:10.20882/adicciones.988.
Azim, T., Bontell, I. y Strathdee, S. A. (2015). Women, drugs and HIV. International Journal of Drug Policy, 26 (Supl. 1), 16-21. doi:10.1016/j.drugpo.2014.09.003.

Baral, S., Logie, C. H., Grosso, A., Wirtz, A. L. y Beyrer, C. (2013). Modified social ecological model: A tool to guide the assessment of the risks and risk contexts of HIV epidemics. BMC Public Health, 13, 482. doi:10.1186/14712458-13-482.

Bourgois, P., Prince, B. y Moss, A. (2004). The everyday violence of hepatitis $\mathrm{C}$ among young women who inject drugs in San Francisco. Human Organization, 63, 253264. doi:10.17730/humo.63.3.h1phxbhrb7m4mlv0.

Chait, L. y Calvo, B. Z. (2005). Mujeres drogodependientes maltratadas: Análisis para la intervención. Trastornos Adictivos, 7, 104-113. doi:10.1016/S1575-0973(0.5)74515-3.

Chohan, B. H., Lavreys, L., Mandaliya, K. N., Kreiss, J. K., Bwayo, J. J., Ndinya-Achola, J. O. y Martin, H. L. (2001). Validation of a modified commercial enzyme-linked immunoassay for detection of human immunodeficiency virus type 1 immunoglobulin $\mathrm{G}$ antibodies in saliva. Clinical and Diagnostic Laboratory Immunology, 8, 346-348. doi:10.1128/CDLI.8.2.346-348.2001.

Decker, M. R., Miller, E., McCauley, H. L., Tancredi, D. J., Anderson, H., Levenson, R. R. y Silverman, J. G. (2014). Recent partner violence and sexual and drug-related STI/HIV risk among adolescent and young adult women attending family planning clinics. Sexually Transmitted Infections, 90, 145-149. doi:10.1136/sextrans-2013-051288.

Deering, K. N., Lyons, T., Feng, C. X., Nosyk, B., Strathdee, S. A., Montaner, J. S. G. y Shannon, K. (2013). Client demands for unsafe sex: The socioeconomic risk environment for HIV among street and off-street sex workers. Journal of Acquired Immune Deficiency Syndromes, 63, 522-531. doi:10.1097/QAI.0b013e3182968d39.

El-Bassel, N., Wechsberg, W. M. y Shaw, S. A. (2012). Dual HIV risk and vulnerabilities among women who use or inject drugs: No single prevention strategy is the answer. Current Opinion in HIV and AIDS, 7, 326-331. doi:10.1097/COH.0b013e3283536ab2.

El-Bassel, N., Shaw, S. A., Dasgupta, A. y Strathdee, S. A. (2014). Drug use as a driver of HIV risks: Re-emerging and emerging issues. Current Opinion in HIV and AIDS, 9, 150-5. doi:10.1097/COH.0000000000000035.

Espelt, A., Mari-Dell'Olmo, M., Penelo, E. y Bosque-Prous, M. (2017). Applied Prevalence Ratio estimation with different Regression models: An example from a cross-national study on substance use research. Adicciones, 29, 105-112. doi:10.20882/adicciones.823.

Espelt, A., Bosque-Prous, M. y Marí-Dell'Olmo, M. (2019). Considerations on the use of Odds Ratio versus Prevalence or Proportion Ratio. Adicciones, 31, 257-259. doi:10.20882/adicciones.1416.

Falcón, C. M. (2006). Invisibilidad y estigmatización del consumo de drogas en las mujeres. En A. García-Mina y M. J. 
Carrasco (Eds.), Diferencias de género en el uso de las drogas (pp. 13-35). España: Universidad Pontificia Comillas.

Folch, C., Esteve, A., Sanclemente, C., Martró, E., Lugo, R., Molinos, S.,... Casabona, J. (2008). Prevalence of human immunodeficiency virus, Chlamydia trachomatis, and Neisseria gonorrhoeae and risk factors for sexually transmitted infections among immigrant female sex workers in Catalonia, Spain. Sexually Transmitted Diseases, 35, 178-183. doi:10.1097/OLQ.0b013e31815a848d.

Gilbert, L., El-Bassel, N., Manuel, J., Wu, E., Go, H., Golder, S.,... Sanders, G. (2006). An integrated relapse prevention and relationship safety intervention for women on methadone: Testing short-term effects on intimate partner violence and substance use. Violence and Victims, 21, 657-672.

Gilbert, L., Raj, A., Hien, D., Stockman, J., Terlikbayeva, A. y Wyatt, G. (2015). Targeting the SAVA (substance abuse, violence and AIDS) syndemic among women and girls: A global review of epidemiology and integrated interventions. Journal of Acquired Immune Deficiency Syndromes, 69 (Supl. 2), 118-27. doi:10.1097/QAI.0000000000000626.

Iversen, J., Page, K., Madden, A. y Maher, L. (2015). HIV, $\mathrm{HCV}$, and health-related harms among women who inject drugs: Implications for prevention and treatment. Journal of Acquired Immune Deficiency Syndromes, 69 (Supl. 2), 176-181. doi:10.1097/QAI.0000000000000659.

Jiménez, A. M., Molina, M. I. S.-M. y García-Palma, M. B. (2014). Gender bias in addictions and their treatment. An overview from the social perspective. Procedia-Social and Behavioral Sciences, 132, 92-99. doi:10.1016/j.sbspro.2014.04.283.

Judd, A., Parry, J., Hickman, M., McDonald, T., Jordan, L., Lewis, K.,... Nelson, M. (2003). Evaluation of a modified commercial assay in detecting antibody to hepatitis $\mathrm{C}$ virus in oral fluids and dried blood spots. Journal of Medical Virology, 71, 49-55. doi:10.1002/jmv.10463.

Li, Y., Marshall, C. M., Rees, H. C., Nunez, A., Ezeanolue, E. E. y Ehiri, J. E. (2014). Intimate partner violence and HIV infection among women: A systematic review and meta-analysis. Journal of the International AIDS Society, 17, 18845. doi:10.7448/IAS.17.1.18845. eCollection 2014.

Lipsky, S., Krupski, A., Roy-Byrne, P., Lucenko, B., Mancuso, D. y Huber, A. (2010). Effect of co-occurring disorders and intimate partner violence on substance abuse treatment outcomes. Journal of Substance Abuse Treatment, 38, 231-244. doi:10.1016/j.jsat.2009.12.005.

Llopis, J. J., Castillo, A., Rebollida, M. y Stocco, P. (2005). Uso de drogas y violencia de género en mujeres adictas en Europa. Claves para su comprensión e intervención. Salud y Drogas, 5, 137-158.

Malinowska-Sempruch, K., Rychkova, O. y Foundations, O. S. (2015). The impact of drug policy on women. New York, NY: Open Society Foundations.

Marshall, B. D. L., Fairbairn, N., Li, K., Wood, E. y Kerr, T. (2008). Physical violence among a prospective cohort of injection drug users: A gender-focused approach. Drug and Alcohol Dependence, 97, 237-246. doi:10.1016/j.drugalcdep.2008.03.028.

Martínez Redondo, P. (2009). Investigación sobre la intervención en drogodependencias y malos tratos a mujeres en la red de atención. Unión de Asociaciones y Entidades de Atención al Drogodependiente, UNAD.

Meyer, J. P., Springer, S. A. y Altice, F. L. (2011). Substance abuse, violence, and HIV in women: A literature review of the syndemic. Journal of Women's Health, 20, 991-1006. doi:10.1089/jwh.2010.2328.

Robertson, A. M., Syvertsen, J. L., Amaro, H., Martinez, G., Rangel, M. G., Patterson, T. L. y Strathdee, S. A. (2014). Can't buy my love: A typology of female sex workers' commercial relationships in the Mexico-U.S. Border Region. Journal of Sex Research, 51, 711-720. doi:10.1080/00 224499.2012.757283.

Ruiz-Olivares, R. y Chulkova, M. (2016). Intervención psicológica en mujeres drogodependientes: Una revisión teórica. Clínica y Salud, 27, 1-6. doi:10.1016/j.clysa.2016.01.001 1130-5274.

Strathdee, S. A., Lozada, R., Martinez, G., Vera, A., Rusch, M., Nguyen, L.,... Patterson, T. L. (2011). Social and structural factors associated with HIV infection among female sex workers who inject drugs in the Mexico-US border region. PloS one, 6, e19048. doi:10.1371/journal. pone.0019048.

Tirado-Muñoz, J., Gilchrist, G., Lligoña, E., Gilbert, L. y Torrens, M. (2015). Intervención grupal para reducir la violencia de género entre consumidoras de drogas. Resultados de un estudio piloto en un centro comunitario de tratamiento de adicciones. Adicciones, 27,168-178. doi:10.20882/adicciones.703.

UN General Assembly. (1993). Declaration on the Elimination of Violence Against Women. Geneva, Switzerland: 85th Plenary Meeting.

UN Women. (2014). A gender perspective on the impact of drug use, the drug trade, and drug control regimes: UN Women policy brief. New York: United Nations Women.

UNAIDS. (2010). Combination HIV Prevention: Tailoring and coordinating biomedical, behavioural and structural strategies to reduce new HIV infections. Geneva, Switzerland: Joint United Nations on HIV/AIDS (UNAIDS).

UNAIDS. (2014). The gap report. Joint United Nations on HIV/AIDS (UNAIDS). Geneva, Switzerland: UNAIDS.

Wagner, K. D., Hudson, S. M., Latka, M. H., Strathdee, S. A., Thiede, H., Mackesy-Amiti, M. E. y Garfein, R. S. (2009). The effect of intimate partner violence on receptive syringe sharing among young female injection drug users: An analysis of mediation effects. AIDS and Behavior, 13, 217-224. doi:10.1007/s10461-007-9309-5.

World Health Organization. (1994). Multi-city study on drug injecting and risk of HIV infection. Programme on Substance abuse-Final Report. Geneva: WHO. 